\title{
Vertical oscillations of an arcade loop in a gravitationally stratified solar corona
}

\author{
M. Gruszecki and K. Murawski \\ Group of Astrophysics and Gravity Theory, Institute of Physics, UMCS, ul. Radziszewskiego 10, 20-031 Lublin, Poland \\ e-mail: marcingruszecki@wp.pl
}

Received 17 December 2007 / Accepted 9 May 2008

\begin{abstract}
Aims. We consider impulsively-generated vertical oscillations of an arcade loop that is embedded in the gravitationally-stratified solar corona.

Methods. Two-dimensional magnetohydrodynamic equations are solved numerically in the limit of an ideal plasma.

Results. The numerical results indicate that the effect of gravity produces a decrease in the wave period and an increase in the attenuation time. This decrease is a consequence of a higher Alfvén speed within the gravitationally-stratified arcade loop, while the weaker attenuation reflects a smaller amount of wave tunneling than in the gravity-free case. These results are reminiscent of those for TRACE data.
\end{abstract}

Key words. magnetohydrodynamics (MHD) - Sun: corona - Sun: oscillations

\section{Introduction}

Magnetic loops harboring gas at temperatures in the range 1-10 MK are the main ingredients of the solar corona. These loops are able to sustain oscillations (Nakariakov \& Verwichte 2005), which were detected by the Transition Region and Coronal Explorer (TRACE) instrument in cool loops with a temperature $T \approx 1 \mathrm{MK}$ and by Solar Ultraviolet Measurements of Emitted Radiation (SUMER) probe board on SOHO in hot loops $(T>6 \mathrm{MK})$. Hot loops were detected to oscillate mainly in a slow magnetoacoustic standing mode (Wang et al. 2002, 2005), while cool loops sustain essentially fast magnetoacoustic kink oscillations, which occur in two polarizations: Aschwanden et al. (2002) and Schrijver et al. (2002) detected horizontal oscillations, while Wang \& Solanki (2004) observed vertical oscillations. Propagating magnetoacoustic waves were also observed in coronal loops (De Moortel et al. 2002a,b; Marsh et al. 2006). De Moortel et al. (2002a) provided a comprehensive overview of geometric and physical properties of longitudinal oscillations in large coronal loops. They demonstrated that intensity oscillations of almost constant period can be present for several consecutive hours. De Moortel et al. (2002b) found that thermal conduction alone could account for the observed attenuation lengths and wavelengths, and, additionally, explain the correlation between propagation period and attenuation length. Marsh et al. (2006) observed oscillations in the sunspot umbral chromosphere and transition region, which are connected to global $\mathrm{p}$-mode oscillations. These $\mathrm{p}$-modes are believed to undergo mode conversion to slow magnetoacoustic waves in regions of strong magnetic fields.

Fast magnetoacoustic oscillations in curved loops have been modeled by a number of authors. For instance, theoretical models of loops in a gravity-free medium were discussed by Smith et al. (1997), Brady \& Arber (2005), Selwa et al. (2006),
Verwichte et al. (2006a), Diáz et al. (2006), Gruszecki et al. (2008) and Terradas et al. (2008). Brady \& Arber (2005) studied the leakage of fast magnetoacoustic kink oscillations. These oscillations were excited by footpoint motion. The leakage was produced by wave tunneling through an evanescent barrier above the coronal loop. In Verwichte et al. (2006a), the coronal loop was modeled by a curved magnetic slab for an equilibrium density given by a piecewise continuous power-law profile. Depending on the value of the power-law index, the wave modes were trapped or they were all subject to lateral wave leakage (upward or downward). Diáz et al. (2006) studied a coronal loop, which was modified by the addition of a density structure along the loop axis. They showed that the frequency and spatial structure of the trapped modes were sensitive to density variations within the loop. Gruszecki et al. (2008) extended the work of Selwa et al. (2006) by inclusion of a dense photosphere-like layer and compared their results with those obtained for the case of line-tying boundary conditions implemented (Selwa et al. 2006). They found that energy leakage into the photosphere-like layer produced stronger wave attenuation. The time evolution of impulsively-generated waves in a coronal arcade was studied by Terradas et al. (2008). The authors showed that the curvature of magnetic-field lines exerted a significant effect on the properties of all magnetohydrodynamics waves.

There are a few papers that discuss the inclusion of gravity in the loop model. For instance, Miyagoshi et al. (2004) pointed out that the amplitude of oscillations decreases exponentially in time as a result of energy transport by fast magnetoacoustic waves. Del Zanna et al. (2005) showed that efficient attenuation of Alfvén oscillations may result from variations in the background density and Alfvén speed along the loop. McLaughlin \& Ofman (2006) discussed transverse oscillations of an active region loop. They found that these oscillations were rapidly 
attenuated and the amplitude of these oscillations decreased as density contrast between loop and corona increased. They also discovered that the high density loop underwent both vertical and horizontal oscillations.

Vertical oscillations of an arcade loop in a gravitationallystratified solar corona have not been studied so far. The main goal of this paper is to study vertical standing kink oscillations of a magnetic loop that is embedded in the solar corona, which is modeled by an isothermal gravitationally-permeated medium. We then compare our results for such corona with those for a gravity-free atmosphere (Gruszecki et al. 2008).

This paper is organized as follows. The numerical model is described in Sect. 2. The numerical results are presented and discussed in Sect. 3. This paper is concluded by a presentation of the main results in Sect. 4.

\section{A numerical model}

Our model system is taken to be composed of a gravitationallystratified plasma that is permeated by a strong magnetic field. We restrict ourselves to the ideal MHD equations:

$\frac{\partial \varrho}{\partial t}+\nabla \cdot(\varrho \boldsymbol{V})=0$

$\varrho \frac{\partial \boldsymbol{V}}{\partial t}+(\varrho \boldsymbol{V} \cdot \nabla) \boldsymbol{V}=-\nabla p+\varrho \boldsymbol{g}+\frac{1}{\mu}(\nabla \times \boldsymbol{B}) \times \boldsymbol{B}$,

$\frac{\partial p}{\partial t}+(\boldsymbol{V} \cdot \nabla) p+\gamma p \nabla \cdot \boldsymbol{V}=0$,

$\frac{\partial \boldsymbol{B}}{\partial t}=\nabla \times(\boldsymbol{V} \times \boldsymbol{B})$,

$\nabla \cdot \boldsymbol{B}=0$,

$p=\frac{k_{\mathrm{B}}}{m} \varrho T$,

where $\gamma=5 / 3$ is the adiabatic index, $\mu$ is the magnetic permeability, $\varrho$ is mass density, $\boldsymbol{V}$ is flow velocity, $p$ is gas pressure, $\boldsymbol{g}=$ $(0,0, g)$ is gravitational acceleration of its value $g=274 \mathrm{~m} \mathrm{~s}^{-2}$, $\boldsymbol{B}$ is magnetic field, $T$ is temperature, $m$ is mean particle mass, and $k_{\mathrm{B}}$ is Boltzmann's constant.

\subsection{Initial configuration}

We assume that, in equilibrium, the magnetic field is force-free and the pressure gradient force is balanced by the gravity, that is

$\frac{1}{\mu}(\nabla \times \boldsymbol{B}) \times \boldsymbol{B}=0$,
$-\nabla p+\varrho \boldsymbol{g}=0$.

We adopt a coronal arcade model that was described by Priest (1982). The coronal arcade is settled in a two-dimensional and motionless environment $(\boldsymbol{V}=0)$. The equilibrium magnetic field is assumed to be current-free $\left(\frac{1}{\mu} \nabla \times \boldsymbol{B}=0\right)$ and is expressed by a magnetic potential $A(x, z)$ as $\boldsymbol{B}=\nabla \times(A \hat{\boldsymbol{y}})$, such that $A(x, z)=B_{0} \Lambda_{\mathrm{B}} \cos \left(x / \Lambda_{\mathrm{B}}\right) \mathrm{e}^{-z / \Lambda_{\mathrm{B}}}$. As a result, components of $\boldsymbol{B}$ are given by

$$
\left[B_{x}, B_{y}, B_{z}\right]=B_{0}\left[\cos \left(x / \Lambda_{\mathrm{B}}\right), 0, \sin \left(x / \Lambda_{\mathrm{B}}\right)\right] \mathrm{e}^{-z / \Lambda_{\mathrm{B}}},
$$

where $B_{0}$ is the magnetic field at the reference level $z=0$ and $\Lambda_{\mathrm{B}}$ is the magnetic scale-height, such that $\Lambda_{\mathrm{B}}=2 L / \pi$, where $L$ is the horizontal half-width of the arcade, chosen to be $L=100 \mathrm{Mm}$.

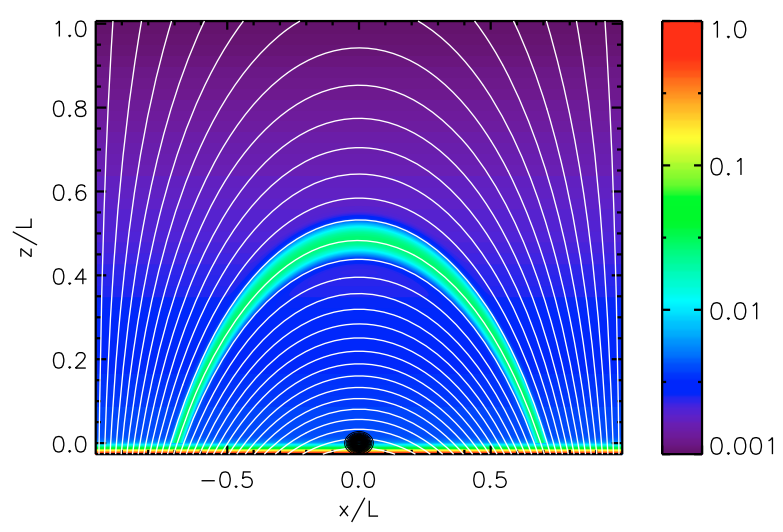

Fig. 1. Initial mass density profile for a loop in a gravitationallystratified medium. Magnetic field lines are shown as solid white lines. The black circle at $(0,0)$ denotes the initial pulse position.

Using the equation of state given in Eq. (6) and the $z$-component of hydrostatic pressure balance indicated by Eq. (8), we express gas pressure and mass density as:

$p(z)=p_{0} \exp \left(-\int_{0}^{z} \frac{\mathrm{d} z^{\prime}}{\tilde{\Lambda}\left(z^{\prime}\right)}\right), \quad \varrho(z)=\frac{p(z)}{g \tilde{\Lambda}(z)}$,

where

$\tilde{\Lambda}(z)=k_{\mathrm{B}} T(z) /(m g)$

is the pressure scale-height, and $p_{0}$ denotes the gas pressure at $z=0$. The limit of $\tilde{\Lambda} \rightarrow \infty$ corresponds to the case of $g=$ 0 (Gruszecki et al. 2008). We adopt a smoothed step-function profile for temperature (del Zanna et al. 2004):

$T(z)=\frac{1}{2}\left(T_{\mathrm{c}}+T_{\mathrm{ph}}\right)+\frac{1}{2}\left(T_{\mathrm{c}}+T_{\mathrm{ph}}\right) \tanh \left(\frac{z-z_{\mathrm{t}}}{z_{\mathrm{w}}}\right)$,

where $T_{\mathrm{ph}}$ denotes photospheric temperature and $T_{\mathrm{c}}$ is the temperature of the solar corona that is separated from the photosphere at the level $z=z_{\mathrm{t}}$ by a transition region of width $z_{\mathrm{w}}=0.025 L$.

Since no analytical expression for a gravitationally-stratified loop is known, we attempt to identify a loop structure numerically. We consider a loop that is embedded in the arcade. A mass density profile of this loop is expressed by

$\varrho_{1}(x, z)=(d+1) \varrho(z) \exp \left[-\frac{\left(z-z_{\mathrm{c}}(x)\right)^{2}}{\sigma^{2}(z)}\right]$

where $\sigma(z)$ is a half-width of the loop and $d$ denotes the mass density contrast between the loop and the ambient medium. The symbol $z_{\mathrm{c}}(x)$ corresponds to a magnetic field line of our choice where $A(x, z)=$ const. We choose $z_{\mathrm{c}}(x)$

$z_{\mathrm{c}}(x)=\frac{1}{2} \Lambda_{\mathrm{B}}\left[\log \left(\frac{\cos \left(x / \Lambda_{\mathrm{B}}\right)}{\cos \left(x_{1} / \Lambda_{\mathrm{B}}\right)}\right)+\log \left(\frac{\cos \left(x / \Lambda_{\mathrm{B}}\right)}{\cos \left(x_{2} / \Lambda_{\mathrm{B}}\right)}\right)\right]$,

where $x_{1}=-0.7 L$ and $x_{2}=x_{1}+\sigma(0)$. The symbol $x_{1}$ corresponds to the external left loop footpoint and $\sigma(0)=2.5 \mathrm{Mm}$ represents the width of the loop at $z=0$. To reproduce accurately an isothermal corona, we initially (at $t=0$ ) modify the gas pressure in the loop region. Such a loop does not correspond to any precise equilibrium. However, after $t \simeq 300 \mathrm{~s}$ it relaxes to a structure that does not evolve significantly with time.

Such a loop does not have a perfectly circular shape (Fig. 1), but its average radius and length can be estimated to 

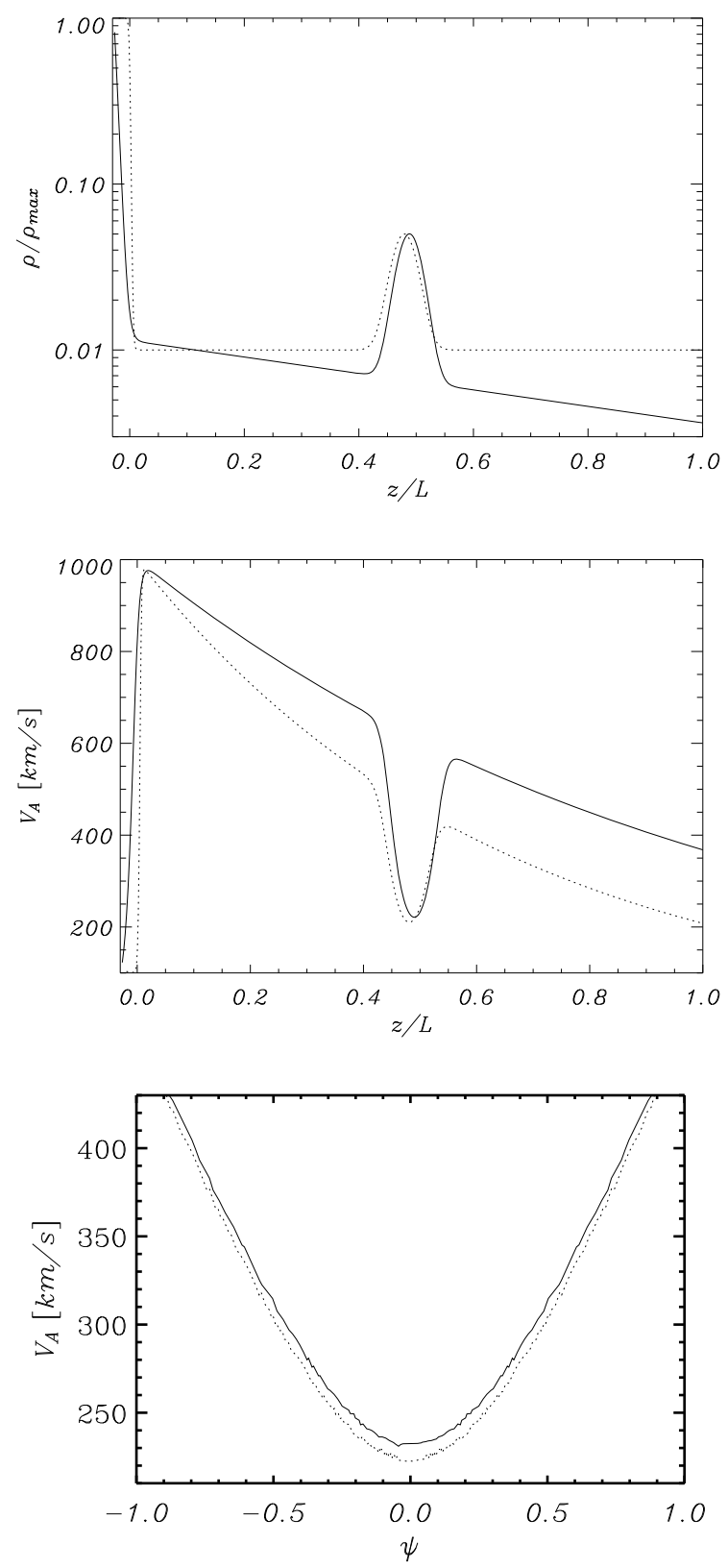

Fig. 2. Mass density along vertical line $x=0$ (top panel), Alfvén speed along $x=0$ (middle panel) and Alfvén speed along the loop (bottom panel). Here, $\psi$ is a spatial coordinate along the loop. Solid lines correspond to the gravitationally-stratified atmosphere with the coronal pressure scale-height, $\Lambda=87 \mathrm{Mm}$, and dotted lines to the gravity-free atmosphere $(\Lambda \rightarrow \infty)$.

be $70 \mathrm{Mm}$ and $190 \mathrm{Mm}$, respectively. These values are close to observationally-determined measurements (Wang \& Solanki 2004). The mass density is enhanced in the loop compared to the ambient medium. We define the mass density contrast to be $d=\varrho_{\mathrm{i}} / \varrho_{\mathrm{e}}=3$, where $\varrho_{\mathrm{i}}$ denotes the mass density within the loop and $\varrho_{\mathrm{e}}$ corresponds to the ambient medium (Fig. 2, top panel). This value of $d$ is based on the observational data of Aschwanden \& Nightingale (2005). We verified that initial states of Eq. (12) for higher values of $d(d \gg 3)$ produce structures that are either Rayleigh-Taylor unstable or require much more time to relax to a structure that does not evolve with time.

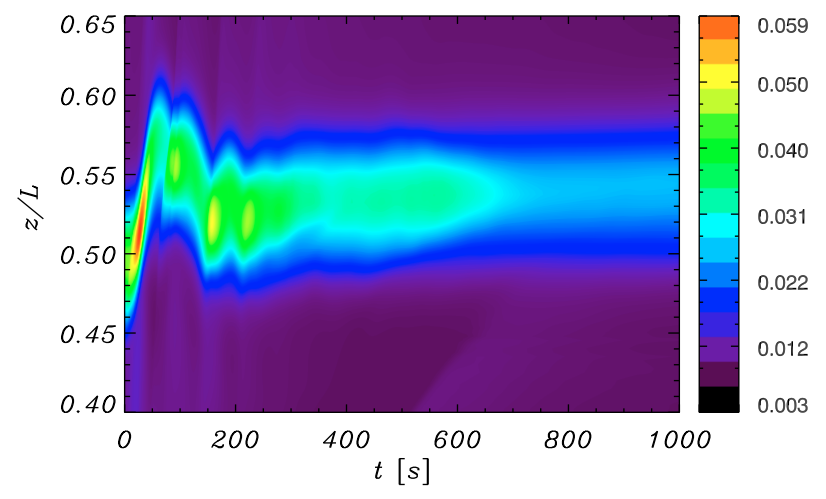

Fig. 3. A typical snapshot of time-signature of the mass density (grey scale; arbitrary units), collected at the loop summit. Here $\Lambda=87 \mathrm{Mm}$ denotes the coronal pressure scale-height and spatial coordinate $z$ and time $t$ are measured in units of $L$ and in seconds, respectively. Mass density is expressed in units of $10^{-15} \mathrm{~kg} \mathrm{~m}^{-3}$.

After the relaxation stage, at $t=322 \mathrm{~s}$, we launch the pulse in vertical component of momentum $\varrho V_{z}$, i.e.

$\varrho V_{z}(x, z, t=0)=A_{\mathrm{m}} \exp \left(-\frac{x^{2}+z^{2}}{w^{2}}\right)$.

Here the amplitude of the initial pulse is chosen as $A_{\mathrm{m}}=$ $0.03 \varrho(0) V_{\mathrm{A}}(0)$, its width $w=0.35 \mathrm{~L}$, and time $t=322 \mathrm{~s}$ is rescaled to $t=0$. A fraction of the momentum is deposited instantly, at $t=0$, at location $(0,0)$ and we then allow the code to develop accordingly. From our experience without gravity, we inferred that it is important to place the pulse at this initial location to be able to excite the fundamental mode of vertical oscillations (Gruszecki et al. 2008).

\section{Numerical results}

To obtain numerical results, we use the code ATHENA, which was developed by Gardiner \& Stone (2005). Athena is a gridbased code for astrophysical plasma dynamics applications. The numerical algorithm that is implemented in the code is based on a higher-order Godunov method with a single-step Eulerian update. Numerical fluxes are computed using a linearized Riemann solver and the divergence-free condition is satisfied with the use of a constraint transport method. To represent a physical region, we use an Eulerian box $(-L, L) \times(-0.02 L, L)$. This box is covered by $400 \times 400$ grid points. We performed grid convergence studies to check the influence of grid size on numerical results. From the obtained results, we inferred that numerical results are not much affected by numerical diffusion and they are well represented by all the chosen grid. We set transparent boundary conditions for perturbed plasma quantities at all boundaries of the simulation region, allowing a wave signal to leave the simulation area freely.

The loop begins to oscillate when fast magnetoacoustic waves, triggered by the initial pulse of Eq. (14), reach its apex. Figure 3 displays the time signature of the mass density along the line $x=0$ in the region close to the apex of the loop. It is clearly visible that the loop exhibits oscillations and does not return to its initial position, displaying an offset. A similar offset was reported by Selwa et al. (2006), who showed that it was produced by the initial pulse energy deposited below the slab apex. This effect is characteristic of 2D geometry, where the region between the curved slab and photosphere layer is a natural wave 


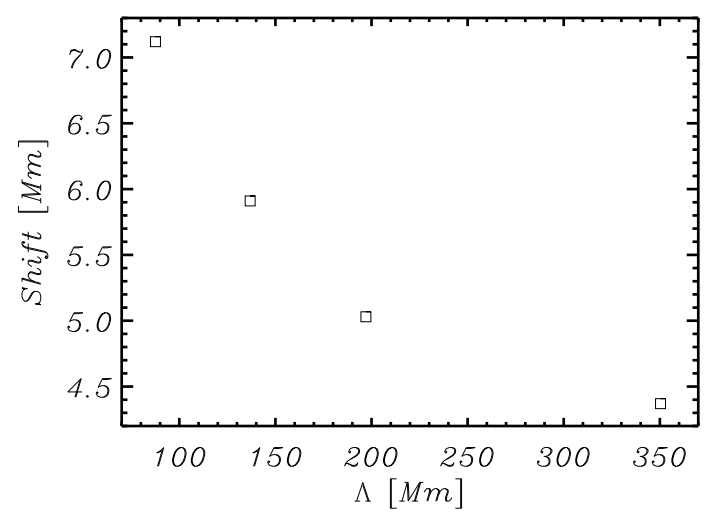

Fig. 4. Maximum displacement of the loop apex versus the coronal pressure scale-height, $\Lambda$.
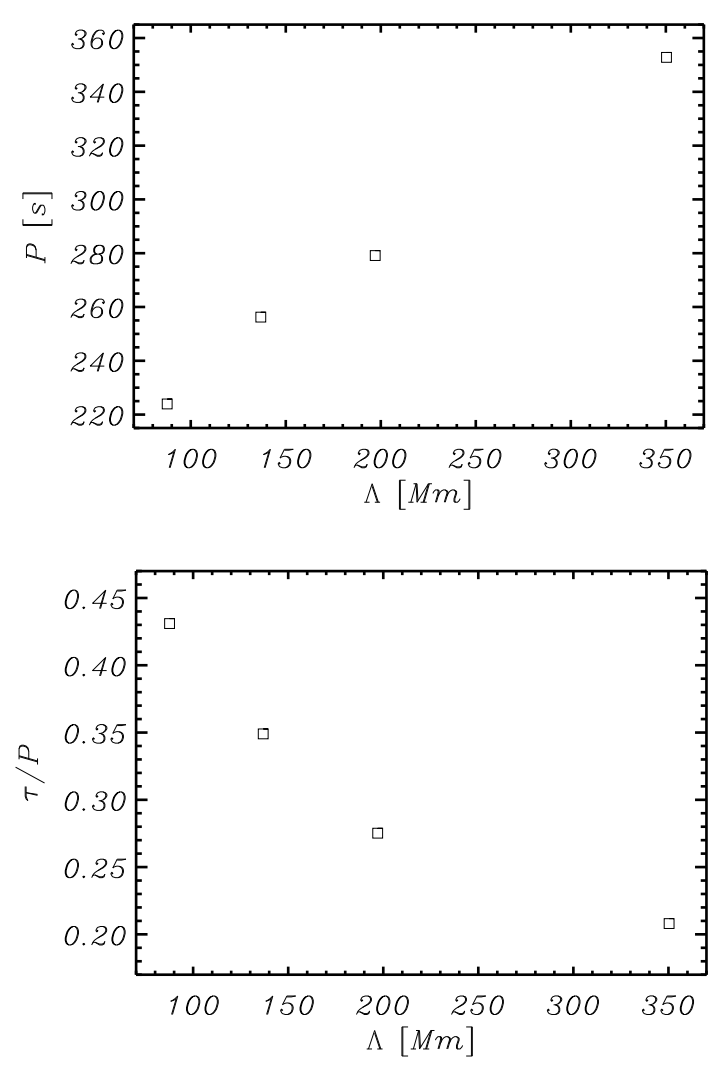

Fig. 5. Wave period $P$ and ratio of attenuation time $\tau$ to the wave period, $\tau / P$, versus $\Lambda$.

cavity (for a detailed discussion see Gruszecki et al. 2008). This offset is larger for a smaller value of the coronal pressure scaleheight, $\Lambda=c_{\mathrm{sc}}^{2} /(\gamma g)$, where $c_{\mathrm{sc}}$ is the sound speed in the solar corona.

Figure 4 shows the maximum displacement of the loop apex during the simulation to measure its dependence on $\Lambda$. We conclude that this displacement declines with $\Lambda$. The evaluated displacement is in the range 4.4-7.1 Mm, which is close to an observational measurement for data at 7.9 Mm (Wang \& Solanki 2004).

Figure 5 (top panel) displays the wave period $P$ versus $\Lambda$. We trace the loop oscillation by following the position of center the apex with time (Fig. 3). We derive both $P$ and the attenuation time $\tau$ of the oscillation by fitting the apex position to the decaying sine function, $\sin (2 \pi t / P) \exp (-t / \tau)$, as described by Selwa et al. (2005). For the straight slab, the wave period can be estimated to be:

$P \simeq \frac{2 l}{\bar{V}_{\mathrm{A}}}$

where $l$ is the initial length of the loop and $\bar{V}_{\mathrm{A}}$ is the average Alfvén speed within the loop. However, although for the curved slab the above formula is not longer valid, it can be used for estimating wave period of the curved slab qualitatively. From Fig. 2 (bottom panel), we infer that $\bar{V}_{\mathrm{A}}$ is larger for a smaller value of $\Lambda$. Applying Eq. (15), we conclude that, for a larger value of $\bar{V}_{\mathrm{A}}$, the wave period is smaller. Such an estimation implies that a positive correlation exists between $P$ and $\Lambda$, which agrees with Fig. 5 (top panel). The observed wave period is equal to $P=234$ s (Wang \& Solanki 2004), which is close to the numerical data for $\Lambda=87 \mathrm{Mm}$ (Fig. 5, top panel).

Figure 5 (bottom panel) illustrates the ratio of attenuation time $\tau$ to the wave period, $\tau / P$, versus $\Lambda$. We conclude that $\tau / P$ declines with increasing $\Lambda$. A larger value of $\Lambda$ produces a stronger attenuation (a smaller attenuation time) of the vertical kink oscillations. Longer wave-period waves are attenuated faster than smaller wave-period wave. Wave attenuation may result from both curvature of magnetic field lines (Selwa et al. 2006) and wave tunneling from the loop into the ambient medium above (Verwichte et al. 2006). Other effects, such as resonance absorption (e.g. Terradas et al. 2007), can also contribute to this scenario. From Fig. 2 (middle panel), we infer that, for a smaller value of $\Lambda$, fast magnetoacoustic waves can tunnel into the ambient medium above, by means of a higher Alfvén speed barrier. As a result, the wave attenuation is smaller for a smaller value of $\Lambda$. The observed value of $\tau / P$ is equal to $\tau / P \simeq 3$ (Wang $\&$ Solanki 2004). From Fig. 5 (bottom panel), we deduce that the numerical values of $\tau / P$ lies in the range $0.2-0.45$. As a result, these values are too small and the oscillations are too strongly attenuated to fit the observational data. However, our results are close to the theoretical estimation of $\tau / P$ by Verwichte et al. (2006).

\section{Summary and discussion}

The results that we have obtained in this paper can be summarized as follows. First, an external pulse that is launched centrally below a loop excites a vertical kink-like mode, which bears many of the properties of the vertical oscillation observed by Wang \& Solanki (2004). The maximum displacement of the loop apex and the ratio of attenuation time to wave period, $\tau / P$, both decay as the coronal pressure scale-height $\Lambda$ increases, while a positive correlation is measured between $P$ and $\Lambda$. While the wave period and the maximum loop displacement both agree with the observational data of Wang \& Solanki (2004), the ratio, $\tau / P$, is found to be too small. We conclude that the present model may require revision. One way to remedy this situation is to include a periodic driver, whose amplitude decays with time. For such a driver, wave attenuation is reduced (Selwa et al. 2008); a similar scenario is expected for a set of pulses. Additionally, the solar atmosphere model requires improvement. For instance, the temperature profile given by Eq. (11) should be replaced by a more realistic profile that exhibits a minimum. Pulse location is important in terms of the excitation of higher-order modes. In particular, a pulse that is initially located off-centrally, e.g. along line $z=-x$ is able to trigger several modes present simultaneously in the loop structure. Variation in pulse duration 
influences $P$ and $\tau$. Such studies are underway and results will be published elsewhere.

In conclusion, we state that more detailed high-resolution observational data such as that from Hinode or TRACE is required to confirm or refute the model.

Acknowledgements. The authors express their cordial thanks to Prof. Sami Solanki and the referee for their comments on an earlier version of this draft. K.M. expresses his gratitude to Dr. Tom Gardiner for his assistance in the implementation of the loop into the code Athena. The magnetohydrodynamics code used in this study was developed at the Princeton University by Tom Gardiner, Jim Stone, Peter Teuben and John Hawley with support of the NSF Information Technology Research program. This work was supported by a grant from the State Committee for Scientific Republic of Poland, with MNiI grant for years 2007-2010.

\section{References}

Aschwanden, M. J., \& Nightingale, R. W. 2005, ApJ, 633, 499

Aschwanden, M. J., de Pontieu, B., Schrijver, C. J., \& Alan, M. 2002, Sol. Phys., 206, 99

Brady, C. S., \& Arber, T. D. 2005, A\&A, 438, 733

del Zanna, L., Schaekens, E., \& Velli, M. 2005, A\&A, 431, 1095

De Moortel, I., Hood, A. W., Ireland, J., \& Walsh, R. W. 2002a, Sol. Phys., 209, 61
De Moortel, I., Hood, A. W., Ireland, J., \& Walsh, R. W. 2002b, Sol. Phys., 209, 89

Diáz, A. J., Oliver, R., \& Ballester, J. L. 2006, ApJ, 645, 766

Gardiner, T. A., \& Stone, J. M. 2005, J. Comput. Phys., 205, 509

Gruszecki, M., Murawski, K., \& McLaughlin, J. A. 2008, A\&A, in press

Marsh, M. S., \& Walsh, R. W. 2006, ApJ, 643, 540

McLaughlin, J. A., \& Ofman, L. 2006, American Geophysical Union, Fall Meeting

Miyagoshi, T., Yokoyama, T., \& Shimojo, M. 2004, Astron. Soc. Jap., 56, 207

Nakariakov, V. M., \& Verwichte, E. 2005, Living Rev. Sol. Phys., 2, 3

Priest, E. R. 1982, Solar Magnetohydrodynamics (Dordrecht: D. Reidel)

Schrijver, C. J., Aschwanden, M. J., \& Title, A. M. 2002, Sol. Phys., 206, 69

Selwa, M., Murawski, K., Solanki, S. K., Wang, T. J., \& Tóth, G. 2005, A\&A, 440,385

Selwa, M., Solanki, S. K., Murawski, K., Wang, T. J., \& Shumlak, U. 2006, A\&A, 454, 653

Selwa, M., Murawski, K., Solanki, S. K., \& Ofman, L. 2008, A\&A, in preparation

Smith, J. M., Roberts, B., \& Oliver, R. 1997, A\&A, 327, 377

Terradas, J., Andries, J., \& Goossens, M. 2007, A\&A, 469, 1135

Terradas, J., Oliver, R., Ballester, J., \& Keppens, R. 2008, ApJ, 675, 875

Verwichte, E., Foullon, C., \& Nakariakov, V. M. 2006, A\&A, 446, 1139

Wang, T. J., \& Solanki, S. K. 2004, A\&A, 421, L33

Wang, T. J., Solanki, S. K., Curdt, W., Innes, D. E., \& Dammasch, I. E. 2002 , 199

Wang, T. J., Solanki, S. K., Innes, D. E., \& Curdt, W. 2005, A\&A, 435, 753 